\title{
Stochastic ordering of Pólya random variables and monotonicity of the Bernstein-Stancu operator for a negative parameter
}

\author{
Florenţa Tripşa' and Nicolae R. Pascu ${ }^{2 *}$ (1)
}

\section{"Correspondence: \\ npascu@kennesaw.edu \\ ${ }^{2}$ Department of Mathematics, \\ Kennesaw State University, Marietta, USA \\ Full list of author information is \\ available at the end of the article}

\begin{abstract}
In the present paper, we prove that the probabilities of the Pólya urn distribution (with negative replacement) satisfy a monotonicity property similar to that of the binomial distribution. As a consequence, we show that the corresponding random variables are stochastically ordered with respect to the parameter giving the initial distribution of the urn. An equivalent formulation of this result shows that the new Bernstein-Stancu-type operator introduced in (Pascu et al. in Proc. Rom. Acad., Ser. A: Math. Phys. Tech. Sci. Inf. Sci. 2019, in press) is a monotone operator.

The proofs are probabilistic in spirit and rely on various inequalities, some of which are of independent interest (e.g., a refined version of the reversed Cauchy-Bunyakovsky-Schwarz inequality or estimates of the error of approximating an integral by the trapezoidal rule).
\end{abstract}

Keywords: Stochastic order; Pólya urn distribution; Bernstein-Stancu operator; Monotone operator

\section{Introduction}

The Pólya urn model (also known as the Pólya-Eggenberger urn model; see [5, 9]) is an experiment in which we observe the number of white balls extracted from an urn containing initially $a$ white balls and $b$ black balls when the extracted ball is replaced in the urn together with $c$ balls of the same color before the next extraction.

Denoting by $X_{n}^{a, b, c}$ the random variable representing the number of white balls obtained in $n \geq 1$ extractions from the urn, it can be shown (e.g., [6]) that the model is well defined (defines a distribution) for $a, b \geq 0$ and $c \in \mathbb{R}$ satisfying $(n-1) c \geq-\min \{a, b\}$, and the distribution is given by

$$
p_{n, k}^{a, b, c}=P\left(X_{n}^{a, b, c}=k\right)=C_{n}^{k} \frac{a^{(k, c)} b^{(n-k, c)}}{1^{(n, c)}}, \quad k \in\{0,1, \ldots, n\},
$$

where $x^{(0, h)}=1$, and $x^{(k, h)}=x(x+h) \cdot \ldots \cdot(x+(k-1) h)$ for $k \geq 1$ denote the rising factorial with increment $h$.

(c) The Author(s) 2019. This article is distributed under the terms of the Creative Commons Attribution 4.0 International License (http://creativecommons.org/licenses/by/4.0/), which permits unrestricted use, distribution, and reproduction in any medium, provided you give appropriate credit to the original author(s) and the source, provide a link to the Creative Commons license, and indicate if changes were made. 
In the present paper, we focus on the case $a=x \in[0,1], b=1-x$, and the minimal choice of the replacement parameter $c=-\min \{x, 1-x\} /(n-1), n>1$. The reason for this choice is twofold. The first is that the limiting (negative) value of $c$ is an interesting problem of study from the probabilistic point of view, with applications in statistics (reliability theory). The second reason relates to the newly introduced operator $R_{n}[7,8]$ defined by

$$
R_{n}(f, x)=P_{n}^{-\min \{x, 1-x\} /(n-1)}(f, x)=E f\left(\frac{1}{n} X_{n}^{x, 1-x,-\min \{x, 1-x\} /(n-1)}\right),
$$

where $P_{n}^{\alpha}$ denotes the classical Bernstein-Stancu operator (see [11] or [3] for a survey on Bernstein-Stancu operators).

The structure of the paper is the following. Section 2 contains some auxiliary results of independent interest, needed in the sequel. In Lemma 1, we prove an interesting inequality, which may be seen as a refined version of a reversed Cauchy-Bunyakovsky-Schwarz inequality (see Remark 2). In Lemma 3, we give bounds for the error of approximation of an integral by trapezoidal rule in terms of the first derivative for the function (which complements the asymptotic error estimates, valid just for large values of the parameter), a useful practical result in numerical analysis. Lemma 4 is a technical result concerning the sign of a certain function, essential for proving our main results.

In Sect. 3, we first prove that the Pólya urn probabilities satisfy a certain monotonicity property with respect to the initial urn distribution (Theorem 5). Using this, in Theorem 7 , we show that the Pólya random variables satisfy a natural stochastic ordering (in terms of reliability theory, this shows that the corresponding survival function is increasing with respect to the parameter). In Theorem 8 , we give an equivalent formulation of this result, which shows that the operator $R_{n}$ is a monotone operator, a property of the classical Bernstein operator (see, e.g., [2]).

\section{Auxiliary results}

We begin with the following auxiliary result of independent interest.

Lemma 1 For integers $n \geq 2$ and $k \in\{1, \ldots, n-1\}$ and positive real numbers $a_{1}, \ldots, a_{n}$, $b_{1}, \ldots, b_{n-k}$ with $\max _{1 \leq i \leq n} a_{i} \leq \min _{1 \leq j \leq n-k} b_{j}$ and $\sum_{i=1}^{n} a_{i}=\sum_{j=1}^{n-k} b_{j}$, we have

$$
\sum_{i=1}^{n} a_{i}^{2}<\sum_{j=1}^{n-k} b_{j}^{2}
$$

Proof Without loss of generality, we may assume that $a_{1} \leq \cdots \leq a_{n} \leq b_{1} \leq \cdots \leq b_{n-k}$.

Note that $\frac{\sum_{i=1}^{n} a_{i}}{n-k}=\frac{\sum_{j=1}^{n-k} b_{j}}{n-k} \geq b_{1} \geq a_{i}$ for $i \in\{1, \ldots, n\}$. Moreover, since $k \geq 1$ and $a_{1}, \ldots, a_{n}>0$, there exists $i \in\{1, \ldots, n\}$ for which $a_{i}<\frac{\sum_{j=1}^{n} a_{j}}{n-k}$ is a strict inequality (otherwise, summing over $i \in\{1, \ldots, n\}$, we would obtain $k=0$ ).

We obtain

$$
\sum_{i=1}^{n} a_{i}^{2}=\sum_{i=1}^{n}\left(a_{i} \cdot a_{i}\right)<\frac{1}{n-k} \sum_{i=1}^{n}\left(a_{i} \sum_{j=1}^{n} a_{j}\right)=\frac{1}{n-k}\left(\sum_{i=1}^{n} a_{i}\right)^{2},
$$


and using the Cauchy-Bunyakovsky-Schwarz inequality, we conclude that

$$
\sum_{i=1}^{n} a_{i}^{2}<\frac{1}{n-k}\left(\sum_{i=1}^{n} a_{i}\right)^{2}=\frac{1}{n-k}\left(\sum_{j=1}^{n-k} b_{j}\right)^{2} \leq \sum_{j=1}^{n-k} b_{j}^{2} .
$$

Remark 2 From inequality (3) we can obtain a reversed Cauchy-Bunyakovsky-Schwarz inequality (see, e.g., [4]) as follows. In the notation of the previous lemma, taking $b_{i}=$ $\frac{1}{n} \sum_{i=1}^{n} a_{i}, i \in\{1, \ldots, n-k\}$, we obtain

$$
\frac{\sum_{i=1}^{n} a_{i}^{2}}{\left(\sum_{i=1}^{n} a_{i}\right)^{2}} \leq \frac{1}{n-k}
$$

which is especially useful when $k \geq 1$ is small. For example, we can take $k=1$ if $a_{n} \leq$ $\frac{1}{n-1} \sum_{i=1}^{n} a_{i}$, the condition which holds in particular if $p \in\{1, \ldots,[\sqrt{A /(A-a)}]\}$ of the numbers $a_{1}, \ldots, a_{n}$ are equal to $a>0$ and $q=n-p \geq 1$ of them are equal to $A>a$.

The Pólya-Szegö inequality (see, e.g., [4, Theorem 5.5]) is the following reversed Cauchy-Bunyakovsky-Schwarz inequality:

$$
\frac{\sum_{i=1}^{n} a_{i}^{2} \sum_{i=1}^{n} b_{i}^{2}}{\left(\sum_{i=1}^{n} a_{i} b_{i}\right)^{2}} \leq \frac{1}{4}\left(\sqrt{\frac{A B}{a b}}+\sqrt{\frac{a b}{A B}}\right)^{2}
$$

where $0<a \leq a_{i} \leq A<\infty$ and $0<b \leq b_{i} \leq B<\infty, i \in\{1, \ldots, n\}$. Taking $b_{1}=\cdots=b_{n}=1$ (thus $b=B=1$ ), it becomes

$$
\frac{\sum_{i=1}^{n} a_{i}^{2}}{\left(\sum_{i=1}^{n} a_{i}\right)^{2}} \leq \frac{1}{4 n}\left(\sqrt{\frac{A}{a}}+\sqrt{\frac{a}{A}}\right)^{2}
$$

for any sequence such that $0<a \leq a_{1} \leq a_{2} \leq \cdots \leq a_{n} \leq A$.

If $A>a(1+2 /(\sqrt{n}-1))$, then the right-hand side of (7) is greater than $\frac{1}{n-1}$, and therefore inequality (5) (with $k=1$ ) improves the Pólya-Szegö inequality (under the hypotheses considered).

We will also need the following auxiliary result, which gives bounds for the error of approximation of an integral by the trapezoidal rule in terms of the first derivative of a function. Before stating the result, we note that such a result is known, but only in the asymptotic case (asymptotic error estimate): denoting by $E_{N}(f)$ the exact error and $\tilde{E}_{N}(f)=$ $\frac{1}{12 N^{2}}\left(f^{\prime}(1)-f^{\prime}(0)\right)$, we have $\lim _{N \rightarrow \infty} E_{N}(f) / \tilde{E}_{N}(f)=1$ (see [1, Sect. 5.1]), whereas our result below shows that $0 \leq E_{N}(f) \leq \frac{1}{4 N^{2}}\left(f^{\prime}(1)-f^{\prime}(0)\right)$ for all $N \geq 1$.

Lemma 3 Suppose $f \in C^{3}([0,1])$ is such that $f, f^{\prime}, f^{\prime \prime}, f^{\prime \prime \prime} \geq 0$ on $[0,1]$. Then for any integer $N \geq 1$, we have

$$
0 \leq \sum_{i=0}^{N} f\left(\frac{i}{N}\right)-N \int_{0}^{1} f(t) d t-\frac{f(0)+f(1)}{2} \leq \frac{f^{\prime}(1)-f^{\prime}(0)}{4 N} .
$$


Figure 1 Trapezoids $T_{i}$ and $T_{i}^{\prime}$ in the proof of Lemma 3. Area $\left(T_{i}^{\prime}\right)$ $\leq \int_{x_{i}}^{x_{i+1}} f(x) d x \leq \operatorname{Area}\left(T_{i}\right)$

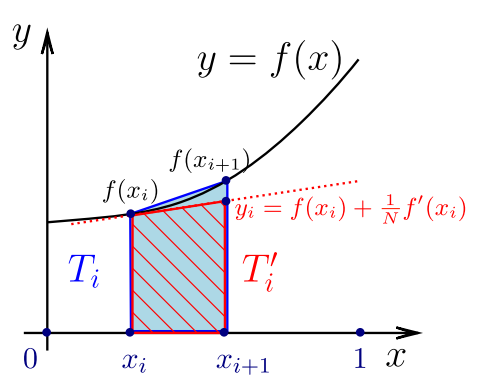

Proof Since $f$ is convex, the sum of the areas of trapezoids $T_{0}, \ldots, T_{N-1}$ (see Fig. 1) is larger than the area under the graph of $f$, and thus

$$
\int_{0}^{1} f(t) d t \leq \sum_{i=0}^{N-1}\left(\frac{f\left(x_{i}\right)+f\left(x_{i+1}\right)}{2} \cdot \frac{1}{N}\right)
$$

where $x_{i}=\frac{i}{N}, i \in\{0,1, \ldots, N\}$, which proves the left inequality in (8).

Since $f$ is convex, the tangent line to the graph of $f$ at $x_{i}$ lies below the graph of $f$, and therefore the sum of the areas of the corresponding trapezoids $T_{i}^{\prime}$ is smaller than the area under $f$ (see Fig. 1). Summing over $i \in\{0,1, \ldots, N-1\}$, we obtain

$$
\sum_{i=0}^{N-1}\left(\frac{f\left(x_{i}\right)+y_{i}}{2} \cdot \frac{1}{N}\right) \leq \int_{0}^{1} f(t) d t
$$

where $y_{i}=f\left(x_{i}\right)+f^{\prime}\left(x_{i}\right) \cdot \frac{1}{N}$, or, equivalently,

$$
N \int_{0}^{1} f(t) d t \geq \sum_{i=0}^{N-1} \frac{f\left(x_{i}\right)+f\left(x_{i}\right)+\frac{f^{\prime}\left(x_{i}\right)}{N}}{2}=\sum_{i=0}^{N-1} f\left(x_{i}\right)+\frac{1}{2 N} \sum_{i=0}^{N-1} f^{\prime}\left(x_{i}\right),
$$

and therefore

$$
\sum_{i=0}^{N} f\left(x_{i}\right) \leq f(1)+\frac{1}{2 N} f^{\prime}(1)+N \int_{0}^{1} f(t) d t-\frac{1}{2 N} \sum_{i=0}^{N} f^{\prime}\left(x_{i}\right)
$$

Using inequality (9) with $f$ replaced by $f^{\prime}$, we obtain

$$
\begin{aligned}
\sum_{i=0}^{N} f\left(x_{i}\right) & \leq f(1)+\frac{1}{2 N} f^{\prime}(1)+N \int_{0}^{1} f(t) d t-\frac{1}{2 N} \sum_{i=0}^{N} f^{\prime}\left(x_{i}\right) \\
& \leq f(1)+\frac{1}{2 N} f^{\prime}(1)+N \int_{0}^{1} f(t) d t-\frac{1}{2 N}\left(N \int_{0}^{1} f^{\prime}(t) d t+\frac{f^{\prime}(0)+f^{\prime}(1)}{2}\right) \\
& =N \int_{0}^{1} f(t) d t+\frac{f(0)+f(1)}{2}+\frac{f^{\prime}(1)-f^{\prime}(0)}{4 N}
\end{aligned}
$$

concluding the proof.

The following technical result is essential for the proof of our main results in the following section. 
Lemma 4 For any integers $n \geq 2$ and $k \in\{0, \ldots, n-1\}$, there exists $x_{n, k} \in\left[\frac{k-1}{n-1}, \frac{k}{n-1}\right]$ such that the function

$$
\varphi_{n, k}(x)=\sum_{i=0}^{n-1} \frac{1}{1-\frac{i}{n-1} x}-\sum_{i=0}^{n-k-1} \frac{1}{1-x-\frac{i}{n-1} x}, \quad x \in\left[0, \frac{n-1}{2 n-k-2}\right),
$$

is positive on $\left(0, x_{n, k}\right)$ and negative on $\left(x_{n, k}, \frac{n-1}{2 n-k-2}\right)$.

Proof Under the hypotheses on $n$ and $k$, it is easy to verify that $\frac{k-1}{n-1}<\frac{k}{n}<\frac{n-1}{2 n-k-2} \leq 1$ (the last inequality is strict if $k<n-1$ ).

If $k=0$, then since $\frac{1}{1-x-\frac{i}{n-1} x}>\frac{1}{1-\frac{i}{n-1} x}$ for $i \in\{0, \ldots, n-1\}$ and $x \in\left(0, \frac{1}{2}\right)$, we have $\varphi_{n, 0}(x)<0$ for $x \in\left(0, \frac{1}{2}\right)$, and the claim holds with $x_{n, 0}=0 \in\left[-\frac{1}{n-1}, 0\right]$.

If $k=n-1$, then we have $\varphi_{n, n-1}(x)=\sum_{i=0}^{n-2} \frac{1}{1-\frac{i}{n-1} x}>0$ for $x \in[0,1)$, and the claim holds with $x_{n, n-1}=1 \in\left[\frac{n-2}{n-1}, 1\right]$.

Assume now that $n>2$ and $k \in\{1, \ldots, n-2\}$. We will first show that if $\varphi_{n, k}(x)=0$, then $\varphi_{n, k}^{\prime}(x)<0$ (note that $\varphi_{n, k}(0)=k>0$ and thus $x \neq 0$ ).

We have

$$
\begin{aligned}
\varphi_{n, k}^{\prime}(x) & =\sum_{i=0}^{n-1} \frac{\frac{i}{n-1}}{\left(1-\frac{i}{n-1} x\right)^{2}}-\sum_{i=0}^{n-k-1} \frac{1+\frac{i}{n-1}}{\left(1-x-\frac{i}{n-1} x\right)^{2}} \\
& =\frac{1}{x}\left(\sum_{i=0}^{n-1} \frac{-\left(1-x \frac{i}{n-1}\right)+1}{\left(1-\frac{i}{n-1} x\right)^{2}}-\sum_{i=0}^{n-k-1} \frac{-\left(1-x\left(1+\frac{i}{n-1}\right)\right)+1}{\left(1-x-\frac{i}{n-1} x\right)^{2}}\right) \\
& =-\frac{1}{x} \varphi_{n, k}(x)+\frac{1}{x}\left(\sum_{i=0}^{n-1} \frac{1}{\left(1-\frac{i}{n-1} x\right)^{2}}-\sum_{i=0}^{n-k-1} \frac{1}{\left(1-x-\frac{i}{n-1} x\right)^{2}}\right) .
\end{aligned}
$$

If $\varphi_{n, k}(x)=0$, then we obtain $\varphi_{n, k}^{\prime}(x)=\frac{1}{x}\left(\sum_{i=0}^{n-1} \frac{1}{\left(1-\frac{i}{n-1} x\right)^{2}}-\sum_{i=0}^{n-k-1} \frac{1}{\left(1-x-\frac{i}{n-1} x\right)^{2}}\right)$, and we are left to prove the implication

$$
\sum_{i=0}^{n-1} \frac{1}{1-\frac{i}{n-1} x}=\sum_{i=0}^{n-k-1} \frac{1}{1-x-\frac{i}{n-1} x} \Rightarrow \sum_{i=0}^{n-1} \frac{1}{\left(1-\frac{i}{n-1} x\right)^{2}}<\sum_{i=0}^{n-k-1} \frac{1}{\left(1-x-\frac{i}{n-1} x\right)^{2}} .
$$

Choosing $a_{i+1}=\frac{1}{1-\frac{i}{n-1} x}, i \in\{0, \ldots, n-1\}$, and $b_{j+1}=\frac{1}{1-x-\frac{j}{n-1} x}, j \in\{0, \ldots, n-k-1\}$, we have $\max _{1 \leq i \leq n} a_{i}=a_{n}=\frac{1}{1-x}=b_{1}=\min _{1 \leq j \leq n-k} b_{j}$, and the implication follows from Lemma 1 , concluding the proof of the claim.

We showed that $\varphi_{n, k}(x)=0$ implies $\varphi_{n, k}^{\prime}(x)<0$. Since $\varphi_{n, k}$ is continuously differentiable, a moment's thought shows that this condition implies that $\varphi_{n, k}$ can change signs at most once on the interval $\left[0, \frac{n-1}{2 n-k-2}\right)$.

Since $\varphi_{n, k}(0)=n-(n-k)=k>0$ and $\lim _{x / \frac{n-1}{2 n-k-2}} \varphi_{n, k}(x)=-\infty$, the function $\varphi_{n, k}$ changes sign on [0, $\left.\frac{n-1}{2 n-k-2}\right)$; let $x_{n, k}$ denote its unique root. We are left to show that $x_{n, k}$ belongs to the specified interval. 
Using Lemma 3 with $N=n-1$ and $f(t)=\frac{1}{1-t x}$, respectively, with $N=n-k-1$ and $f(t)=\frac{1}{1-x-t \frac{n-k-1}{n-1} x}$, we obtain:

$$
\begin{aligned}
\varphi_{n, k}(x) \leq & \left((n-1) \int_{0}^{1} \frac{1}{1-t x} d t+\frac{1+\frac{1}{1-x}}{2}+\frac{\frac{x}{(1-x)^{2}}-x}{4(n-1)}\right) \\
& -\left((n-k-1) \int_{0}^{1} \frac{1}{1-x-t x \frac{n-k-1}{n-1}} d t+\frac{\frac{1}{1-x}+\frac{1}{1-x-\frac{n-k-1}{n-1} x}}{2}\right) \\
= & \left(-\frac{n-1}{x} \ln (1-x)+\frac{1+\frac{1}{1-x}}{2}+\frac{\frac{x}{(1-x)^{2}}-x}{4(n-1)}\right) \\
& -\left(-\frac{n-1}{x} \ln \frac{1-x-\frac{n-k-1}{n-1} x}{1-x}+\frac{\frac{1}{1-x}+\frac{1}{1-x-\frac{n-k-1}{n-1} x}}{2}\right) \\
= & \frac{1}{2}\left(1-\frac{1}{1-x-\frac{n-k-1}{n-1} x}\right)+\frac{x^{2}(2-x)}{4(n-1)(1-x)^{2}}+\frac{n-1}{x} \ln \frac{1-x-\frac{n-k-1}{n-1} x}{(1-x)^{2}} .
\end{aligned}
$$

In particular, for $x=\frac{k}{n-1}$, we obtain $\varphi_{n, k}\left(\frac{k}{n-1}\right) \leq \frac{k(2 n-k-2)\left(k-2(n-1)^{2}\right)}{4(n-1)^{2}(n-k-1)^{2}}<0$, which shows that $x_{n, k}<\frac{k}{n-1}$.

To obtain the lower bound for $x_{n, k}$, first note that for $k=1$, the claim is trivial $\left(\varphi_{n, 1}(0)=\right.$ $1>0$, and thus $\left.x_{n, 1}>0\right)$, so we may assume that $k \in\{2, \ldots, n-1\}$.

Using again Lemma 3 with the same choices as before, we obtain

$$
\begin{aligned}
& \varphi_{n, k}(x) \geq\left((n-1) \int_{0}^{1} \frac{1}{1-t x} d t+\frac{1+\frac{1}{1-x}}{2}\right)-\left((n-k-1) \int_{0}^{1} \frac{1}{1-x-t x \frac{n-k-1}{n-1}} d t\right. \\
& \left.+\frac{\frac{1}{1-x}+\frac{1}{1-x-\frac{n-k-1}{n-1} x}}{2}+\frac{\frac{\frac{n-k-1}{n-1} x}{\left(1-x-\frac{n-k-1}{n-1} x\right)^{2}}-\frac{\frac{n-k-1}{n-1} x}{(1-x)^{2}}}{4(n-k-1)}\right) \\
& =\left(-\frac{n-1}{x} \ln (1-x)+\frac{1+\frac{1}{1-x}}{2}\right)-\left(-\frac{n-1}{x} \ln \frac{1-x-\frac{n-k-1}{n-1} x}{1-x}\right. \\
& \left.+\frac{\frac{1}{1-x}+\frac{1}{1-x-\frac{n-k-1}{n-1} x}}{2}+\frac{\frac{\frac{n-k-1}{n-1} x}{\left(1-x-\frac{n-k-1}{n-1} x\right)^{2}}-\frac{\frac{n-k-1}{n-1} x}{(1-x)^{2}}}{4(n-k-1)}\right) \\
& =\frac{1}{2}\left(1-\frac{1}{1-x-\frac{n-k-1}{n-1} x}\right)-\left(\frac{1}{\left(1-x-\frac{n-k-1}{n-1} x\right)^{2}}-\frac{1}{(1-x)^{2}}\right) \frac{x}{4(n-1)} \\
& +\frac{n-1}{x} \ln \frac{1-x-\frac{n-k-1}{n-1} x}{(1-x)^{2}} \text {. }
\end{aligned}
$$

To simplify the following computation, denote $A=\left(\frac{n-k}{n-1}\right)^{2}, B=\frac{k-1}{(n-1)^{2}}$, and $C=\frac{A}{B}=\frac{(n-k)^{2}}{k-1}$. For $x=\frac{k-1}{n-1}$, the inequality becomes

$$
\begin{aligned}
\varphi_{n, k}\left(\frac{k-1}{n-1}\right) & \geq \frac{1}{2}\left(1-\frac{1}{A+B}\right)-\left(\frac{1}{(A+B)^{2}}-\frac{1}{A^{2}}\right) \frac{B}{4}+\frac{1}{B} \ln \left(\frac{A+B}{A}\right) \\
& =\frac{1}{2}+\frac{1}{B}\left(-\frac{1}{2(1+C)}-\frac{1}{4(1+C)^{2}}+\frac{1}{4 C^{2}}+\ln \left(1+\frac{1}{C}\right)\right) .
\end{aligned}
$$


Since

$$
\begin{aligned}
& \frac{d}{d C}\left(-\frac{1}{2(1+C)}-\frac{1}{4(1+C)^{2}}+\frac{1}{4 C^{2}}+\ln \left(1+\frac{1}{C}\right)\right) \\
& \quad=\left(\frac{1}{2(1+C)^{2}}-\frac{1}{C(1+C)}\right)+\left(\frac{1}{2(1+C)^{3}}-\frac{1}{2 C^{3}}\right)<0
\end{aligned}
$$

and $\lim _{C \rightarrow \infty}\left(-\frac{1}{2(1+C)}-\frac{1}{4(1+C)^{2}}+\frac{1}{4 C^{2}}+\ln \left(1+\frac{1}{C}\right)\right)=0$, we conclude that $\left(-\frac{1}{2(1+C)}-\frac{1}{4(1+C)^{2}}+\right.$ $\left.\frac{1}{4 C^{2}}+\ln \left(1+\frac{1}{C}\right)\right)>0$ for all $C>0$.

From this and from the previous inequality we obtain $x_{n, k}>\frac{k-1}{n-1}$, concluding the proof.

\section{Main results}

We can now prove the first main result.

Theorem 5 For arbitrarily fixed integers $n \geq 2$ and $k \in\{0,1, \ldots, n\}$, the probability $p_{n, k}(x)=p_{n, k}^{x, 1-x,-\min \{x, 1-x\} /(n-1)}$ given by (1) increases for $x \in\left[0, x_{n, k}^{*}\right]$ and decreases for $x \in$ $\left[x_{n, k}^{*}, 1\right]$, where

$$
x_{n, k}^{*}= \begin{cases}x_{n, k} & \text { if } k \leq \frac{n-1}{2}, \\ \frac{1}{2} & \text { if } \frac{n-1}{2}<k<\frac{n+1}{2}, \\ 1-x_{n, n-k} & \text { if } k \geq \frac{n+1}{2}\end{cases}
$$

and $x_{n, k} \in\left[\frac{k-1}{n-1}, \frac{k}{n-1}\right]$ is given by Lemma 4 .

Proof First, note that $p_{n, 0}(x)=\prod_{i=0}^{n-1} \frac{(1-x-i \min \{x, 1-x\} /(n-1))}{1-i \min \{x, 1-x\} /(n-1)}$ is a decreasing function of $x \in[0,1]$ (each factor decreases in $x$ ), and, similarly, $p_{n, n}(x)=\prod_{i=0}^{n-1} \frac{(x-i \min \{x, 1-x\} /(n-1))}{1-i \min \{x, 1-x\} /(n-1)}$ is an increasing function of $x \in[0,1]$ (each factor increases in $x$ ). The claim of the theorem therefore holds in the cases $k=0$ and $k=n\left(x_{n, 0}^{*}=x_{n, 0}=0\right.$ and $x_{n, n}^{*}=1-x_{n, 0}=1$, respectively), and we can assume that $k \in\{1, \ldots, n-1\}$.

For $x \in(0,1 / 2)$, in the notation of Lemma 4 , we have

$$
\begin{aligned}
\frac{d}{d x} \ln p_{n, k}(x)= & \frac{d}{d x}\left(\ln C_{n}^{k}+\sum_{i=0}^{k-1} \ln \left(x-\frac{i}{n-1} x\right)\right. \\
& \left.+\sum_{i=0}^{n-k-1} \ln \left(1-x-\frac{i}{n-1} x\right)-\sum_{i=0}^{n-k-1} \ln \left(1-\frac{i}{n-1} x\right)\right) \\
= & \frac{k}{x}+\sum_{i=0}^{n-k-1} \frac{-\left(1+\frac{i}{n-1}\right)}{1-x-\frac{i}{n-1} x}-\sum_{i=0}^{n-1} \frac{-\frac{i}{n-1}}{1-\frac{i}{n-1} x} \\
= & \frac{k}{x}+\frac{1}{x} \sum_{i=0}^{n-k-1} \frac{1-\left(1+\frac{i}{n-1}\right) x-1}{1-x-\frac{i}{n-1} x}-\frac{1}{x} \sum_{i=0}^{n-1} \frac{1-\frac{i}{n-1} x-1}{1-\frac{i}{n-1} x} \\
= & \frac{k}{x}+\frac{n-k}{x}-\frac{1}{x} \sum_{i=0}^{n-k-1} \frac{1}{1-x-\frac{i}{n-1} x}-\frac{n}{x}+\frac{1}{x} \sum_{i=0}^{n-1} \frac{1}{1-\frac{i}{n-1} x} \\
= & \frac{1}{x} \varphi_{n, k}(x),
\end{aligned}
$$


and a similar computation shows

$$
\frac{d}{d x} \ln p_{n, k}(x)=-\frac{1}{1-x} \varphi_{n, n-k}(1-x), \quad x \in(1 / 2,1)
$$

(alternatively, to derive this, we can use the relation $p_{n, k}(x)=p_{n, n-k}(1-x)$, valid for $x \in$ $[0,1], n \geq 2$, and $k \in\{0,1, \ldots, n\})$.

It remains to show that the information about the sign of $\varphi_{n, k}(x)$ given by Lemma 4 translates into the monotonicity of $p_{n, k}(x)$ indicated in the statement of the theorem.

Note that by Lemma 4 we have

$$
x_{n, k} \in\left[\frac{k-1}{n-1}, \frac{k}{n-1}\right] \text { for all } n \geq 2 \text { and } k \in\{1, \ldots, n-1\} \text {. }
$$

If $\frac{k}{n-1} \leq \frac{1}{2}$, then Lemma 4 and (12) show that $p_{n, k}$ increases on $\left[0, x_{n, k}\right]$ and decreases on $\left[x_{n, k}, 1 / 2\right]$ (note that $x_{n, k} \leq \frac{1}{2}$ by (14) in this case). Since $x_{n, n-k} \geq \frac{n-k-1}{n-1}=1-\frac{k}{n-1} \geq \frac{1}{2}$, the function $\varphi_{n, n-k}(x)$ is positive for $x \in[0,1 / 2] \subset\left[0, x_{n, n-k}\right]$, and from (13) it follows that $p_{n, k}$ decreases on $[1 / 2,1]$. Since $p_{n, k}$ is a continuous function on $[0,1]$, it follows that $p_{n, k}$ increases on $\left[0, x_{n, k}\right]$ and decreases on $\left[x_{n, k}, 1\right]$, and therefore the claim of the theorem holds in this case with $x_{n, k}^{*}=x_{n, k}$.

If $\frac{k-1}{n-1} \geq \frac{1}{2}$, then Lemma 4 and (12) show that $p_{n, k}$ increases on $[0,1 / 2] \subset\left[0, x_{n, k}\right]$ (note that $x_{n, k} \geq \frac{1}{2}$ by (14) in this case). Since $x_{n, n-k} \leq \frac{n-k}{n-1}=1-\frac{k-1}{n-1} \leq \frac{1}{2}$, the function $\varphi_{n, n-k}(x)$ is positive for $x \in\left[0, x_{n, n-k}\right] \subset[0,1 / 2]$ and negative for $x \in\left[x_{n, n-k}, 1 / 2\right]$, and from (13) it follows that $p_{n, k}$ increases on $\left[\frac{1}{2}, 1-x_{n, n-k}\right]$ and decreases on $\left[1-x_{n, n-k}, 1\right]$. Since $p_{n, k}(x)$ is a continuous function of $x \in[0,1]$, it follows that $p_{n, k}$ increases on $\left[0,1-x_{n, n-k}\right]$ and decreases on $\left[1-x_{n, n-k}, 1\right]$, and therefore the claim of the theorem holds in this case with $x_{n, k}^{*}=1-x_{n, n-k}$.

We are left to consider the case $\frac{k-1}{n-1}<\frac{1}{2}<\frac{k}{n-1}$ or, equivalently, $\frac{n-1}{2}<k<\frac{n-1}{2}+1$. If $n$ is odd, then the previous double inequality is not satisfied for any integer $k$, so assume that $n=2 m$ is even. The previous double inequality gives $m-\frac{1}{2}<k<m+\frac{1}{2}$, which is satisfied only for $k=m$. We have

$$
\sum_{i=0}^{2 m-1} \frac{1}{1-\frac{i}{n-1} \cdot \frac{1}{2}}>\sum_{j=0}^{m-1}\left(\frac{1}{1-\frac{2 j}{n-1} \cdot \frac{1}{2}}+\frac{1}{1-\frac{2 j}{n-1} \cdot \frac{1}{2}}\right)=2 \sum_{j=0}^{m-1} \frac{1}{1-\frac{j}{n-1}},
$$

and therefore

$$
\varphi_{2 m, m}\left(\frac{1}{2}\right)=\sum_{i=0}^{2 m-1} \frac{1}{1-\frac{i}{n-1} \cdot \frac{1}{2}}-\sum_{j=0}^{m-1} \frac{1}{\frac{1}{2}-\frac{j}{n-1} \cdot \frac{1}{2}}>0 .
$$

Since $\varphi_{n, k}=\varphi_{2 m, m}=\varphi_{n, n-k}$ in this case, the previous inequality shows that $x_{n, k}=x_{2 m, m}=$ $x_{n, n-k}>\frac{1}{2}$ (thus $\varphi_{n, k}=\varphi_{n, n-k}$ are positive on $\left(0, \frac{1}{2}\right]$ ). Using (12) and (13), we conclude that $p_{n, k}$ increases on $\left[0, \frac{1}{2}\right]$ and decreases on $\left[\frac{1}{2}, 1\right]$, and thus the claim of the theorem holds with $x_{n, k}^{*}=\frac{1}{2}$ in this case, concluding the proof.

Remark 6 Since $p_{n, k}(x)=p_{n, n-k}(1-x)$ for $x \in[0,1]$, from the previous theorem it follows that $x_{n, k}^{*}=1-x_{n, n-k}^{*}$ for all $n \geq 2$ and $k \in\{0, \ldots, n\}$. 
Moeover, note that since $x_{n, k} \in\left[\frac{k-1}{n-1}, \frac{k}{n-1}\right]$, from (11) it follows that we also have $x_{n, k}^{*} \in$ $\left[\frac{k-1}{n-1}, \frac{k}{n-1}\right]$ for all $n \geq 2$ and $k \in\{0,1, \ldots, n\}$.

We are now ready to prove the main result. Recall that a random variable $X$ is smaller than a random variable $Y$ in the usual stochastic order (denoted $X \leq_{\text {st }} Y$; see, e.g., [10]) if the corresponding distribution functions $F_{X}$ and $F_{Y}$ satisfy $F_{X}(x) \geq F_{Y}(x)$ for all $x \in \mathbb{R}$.

Theorem 7 For any $n \geq 2$, the random variables $X_{n}^{x, 1-x,-\min \{x, 1-x\} /(n-1)}$ with Pólya urn distribution given by (1) and parameter $x \in[0,1]$ satisfy the following stochastic ordering:

$$
X_{n}^{x, 1-x,-\min \{x, 1-x\} /(n-1)} \leq_{\mathrm{st}} X_{n}^{y, 1-y,-\min \{x, 1-y\} /(n-1)}, \quad 0 \leq x \leq y \leq 1 .
$$

Proof Fix $n \geq 2$ and denote by $F_{x}$ the distribution function of the random variable $X_{n}^{x, 1-x,-\min \{x, 1-x\} /(n-1)}, x \in[0,1]$. To prove the claim, it suffices to show that, for any $k \in$ $\{0,1, \ldots, n\}, F_{x}(k)$ is a decreasing function of $x \in[0,1]$, and we will prove this inductively on $k$.

Since $F_{x}(0)=P\left(X_{n}^{x, 1-x,-\min \{x, 1-x\} /(n-1)}=0\right)=p_{n, 0}(x)$ is a decreasing function of $x \in[0,1]$ (by Theorem 5), the claim holds for $k=0$.

Assume now that the claim is true for $k-1$, that is, $F_{x}(k-1)$ is decreasing in $x \in[0,1]$.

Theorem 5 shows that $p_{n, k}(x)$ is a decreasing function of $x \in\left[x_{n, k}^{*}, 1\right]$, and therefore $F_{x}(k)=F_{x}(k-1)+p_{n, k}(x)$ is decreasing for $x \in\left[x_{n, k}^{*}, 1\right]$.

Considering now $x \in\left[0, x_{n, k}^{*}\right]$, we observe that

$$
F_{x}(k)=\sum_{i=0}^{k} p_{n, i}(x)=1-\sum_{i=k+1}^{n} p_{n, n-i}(1-x)=1-\sum_{i=0}^{n-k-1} p_{n, i}(1-x) .
$$

Using Remark 6, we obtain $x_{n, k}^{*}+x_{n, n-k-1}^{*} \leq \frac{k}{n-1}+\frac{n-k-1}{n-1}=1$, and it follows that, for $x \in$ $\left[0, x_{n, k}^{*}\right]$, we have

$$
1-x \geq 1-x_{n, k}^{*} \geq x_{n, n-k-1}^{*} \geq x_{n, i}^{*}, \quad i \in\{0,1, \ldots, n-k-1\},
$$

since by Remark 6 we have $x_{n, i}^{*} \leq \frac{i}{n-1} \leq x_{n, i+1}^{*}$ for $i \in\{0,1, \ldots n-1\}$.

Using (15) and (16), together with the monotonicity of $p_{n, i}$ given by Theorem 5 , it follows that $F_{x}(k)$ is also decreasing for $x \in\left[0, x_{n, k}^{*}\right]$, concluding the proof of the theorem.

It is known (e.g., [10], p. 4) that the stochastic comparison $X \leq_{\text {st }} Y$ is equivalent to $E f(X) \leq E f(Y)$ for all increasing functions $f$ for which the expectations exist.

Using this and definition (2) of the operator $R_{n}$, we can restate the theorem as follows.

Theorem 8 The operator $R_{n}$ defined by (2) is a monotone operator, that is, if $f:[0,1] \rightarrow \mathbb{R}$ is increasing (decreasing), then $R_{n}(f, \cdot):[0,1] \rightarrow \mathbb{R}$ is also increasing (decreasing). 
Competing interests

The authors declare that they have no competing interests.

\section{Authors' contributions}

Not applicable (this is a joint work of the authors, and it is difficult to pinpoint the exact contribution of each author). All authors read and approved the final manuscript.

\section{Author details}

${ }^{1}$ Faculty of Mathematics and Computer Science, Transilvania University of Braşov, Braşov, Romania. ${ }^{2}$ Department of Mathematics, Kennesaw State University, Marietta, USA.

\section{Publisher's Note}

Springer Nature remains neutral with regard to jurisdictional claims in published maps and institutional affiliations.

Received: 28 September 2018 Accepted: 18 February 2019 Published online: 26 February 2019

\section{References}

1. Atkinson, K.E.: An Introduction to Numerical Analysis, 2nd edn. Wiley, New York (1989)

2. Bustamante, J.: Bernstein Operators and Their Properties. Springer, Cham (2017)

3. Della Vecchia, B.: On the approximation of functions by means of the operators of D. D. Stancu. Stud. Univ. Babeş-Bolyai, Math. 37(1), 3-36 (1992)

4. Dragomir, S.S.: A survey on Cauchy-Bunyakovsky-Schwarz type discrete inequalities. J. Inequal. Pure Appl. Math. 4(3), Article 63, 142 pp. (2003)

5. Eggenberger, F., Pólya, G.: Über die Statistik verketteter Vorgänge. Z. Angew. Math. Mech. 3, 279-289 (1923)

6. Johnson, N.L., Kotz, S.: Urn Models and Their Application. An Approach to Modern Discrete Probability Theory. Wiley, New York (1977)

7. Pascu, M.N., Pascu, N.R., Tripşa, F.: An error estimate for a Bernstein-Stancu operator with negative parameter. Results Math. (2019). https://doi.org/10.1007/s00025-019-0968-0

8. Pascu, M.N., Pascu, N.R., Tripşa, F.: A new Bernstein-Stancu type operator with negative replacement. Proc. Rom. Acad., Ser. A: Math. Phys. Tech. Sci. Inf. Sci. (2019, in press). Arxiv preprint https://arxiv.org/abs/1710.08818, Accessed 28 March 2018

9. Pólya, G.: Sur quelques points de la théorie des probabilités. Ann. Inst. Henri Poincaré 1, 117-161 (1931)

10. Shaked, M., Shanthikumar, J.G.: Stochastic Orders. Springer, New York (2007)

11. Stancu, D.D.: Approximation of functions by a new class of linear polynomial operators. Rev. Roum. Math. Pures Appl. 13, 1173-1194 (1968)

\section{Submit your manuscript to a SpringerOpen ${ }^{\circ}$ journal and benefit from:}

- Convenient online submission

- Rigorous peer review

- Open access: articles freely available online

- High visibility within the field

- Retaining the copyright to your article 